\title{
Educating and engaging junior doctors
}

\author{
Author: Fasihul Khan
}

\section{Aims}

To improve the educational experience of trainees in a busy tertiary cardio-respiratory centre.

\section{Methods}

As NHS pressures exponentially rise, there is a growing risk of training being compromised. Alongside this, there seems to be increasing junior doctor apathy towards speaking out with a general lack of engagement. As the chief registrar in a busy tertiary hospital, I have concentrated on a number of interventions to attempt to improve this.

A monthly junior doctor forum, with free lunch, attended by consultants and senior managers has been introduced and welcomed. The forum has encouraged engagement providing a safe platform for trainees to voice their concerns. Coupled with this, a weekly hospital breakfast club, open to all, regardless of grade and specialty, has been set up. As well as providing breakfast, interesting cases are discussed paving the way for case reports, and insightful cross-specialty discussions. More importantly perhaps, it enables doctors to come together away from the busy clinical environment and provide support to one another. A more united cohesive workforce is undoubtedly more efficient, resulting in enhanced patient care. Junior doctor awards have also been introduced as part of the breakfast club with nominations and winners announced monthly.

Improving education has been another area of focus with various interventions. While independence in practical procedures is considered necessary, opportunities can often be scarce and dependent on multiple factors. We therefore set up a procedural bleep held on a rotational basis. Registrars performing procedures were asked to bleep the bleep-holder who then observed, assisted or performed the procedure under supervision, resulting in frequent procedural opportunities for all trainees.

Alongside this, simulation training has been developed and incorporated into our local induction, whereby junior trainees are able to lead in the assessment and management of simulated acutely unwell patients. Senior clinicians, who can be called for support, mimicking real life scenarios, facilitate the sessions.

For more senior trainees, a 'bring the clinic to the registrar' scheme has been developed. Consultants bring patient notes from their clinic and present to the group of registrars. This is followed by

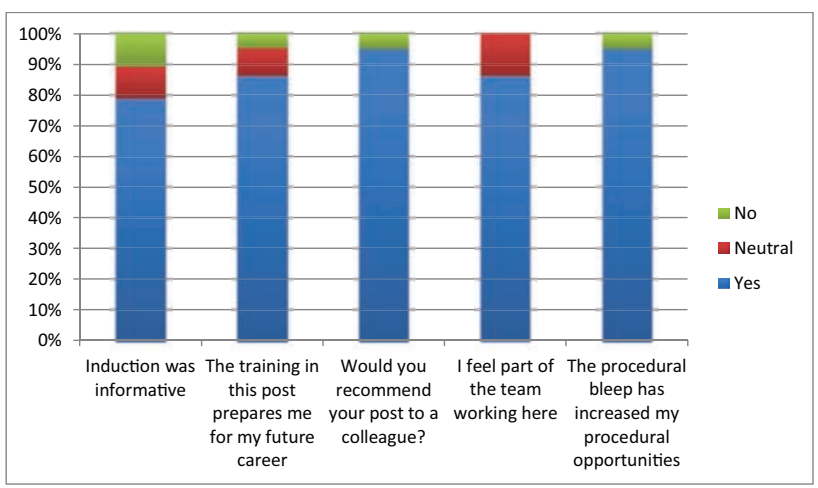

Fig 1. Summary of junior doctor survey results.

insightful discussions around differentials, investigation pathways, management and a review of the evidence.

\section{Results}

In our most recent survey, $95 \%$ of trainees ( 20 out of 21 ) said they would recommend their post to a colleague (Fig 1). Ninety-five per cent felt holding the procedural bleep in an assigned manner increased their exposure to procedures.

\section{Conclusion}

These interventions have been well-received and have raised the educational atmosphere of our hospital.

\section{Conflict of interest statement}

Nil to declare. 